\title{
Effectiveness of Underwater Endoscopic Submucosal Dissection for a Superficial Cervical Esophageal Cancer
}

\author{
Sho Sasaki , Jun Nishikawa' ${ }^{2}$ Kazuhiro Yamamoto ${ }^{1}$ and Isao Sakaida ${ }^{3}$ \\ ${ }^{1}$ Department of Gastroenterology, Saiseikai Yamaguchi Hospital, Yamaguchi, Department of ${ }^{2}$ Laboratory Science, ${ }^{3}$ Gastroenterology \\ and Hepatology, Yamaguchi University Graduate School of Medicine, Yamaguchi, Japan
}

Currently, the underwater endoscopic mucosal resection method is used in colorectal and duodenal lesions because the mucosa and submucosa float into the lumen moving away from the muscularis propria after the lumen is filled with water. ${ }^{1,2}$ We performed underwater endoscopic submucosal dissection (ESD) for a cervical esophageal lesion.

An 81-year-old man with a history of drinking and smoking underwent endoscopy that revealed a superficial esophageal cancer involving the one third of the lumen in the cervical esophagus (Fig. 1A, B). Because of the high risk of aspiration, ESD was performed under general anesthesia and tracheal intubation. A GIF-Q260J endoscope, 1.5-mm Dual Knife, and IT knife nano (all; Olympus, Tokyo, Japan) were used.

Since the lumen was narrow owing to natural constriction, a good view could not be secured under $\mathrm{CO}_{2}$ insufflation. Therefore, we tried the "underwater" method. As the lumen expanded after filling with $0.9 \%$ saline solution using a water pump (OFP-2; Olympus), it was possible to secure a visual field (Fig. 2A). Subsequently, the submucosal layer floated to the surface, and the circumferential incision and submucosal dissection were completed easily and quickly (Fig. 2B, C). It took 70 minutes for the ESD procedure. The pathological results were squamous cell carcinoma, depth of lamina propria,

Received: January 22, 2020 Revised: February 24, 2020

Accepted: March 11, 2020

Correspondence: Sho Sasaki

Department of Gastroenterology, Saiseikai Yamaguchi Hospital, Midorimachi 2-11, Yamaguchi 753-8517, Japan

Tel: +81-083-901-6111, Fax: +81-083-921-0714, E-mail: yudaon@yahoo.co.jp

ORCID: https://orcid.org/0000-0003-4043-5562

(c) This is an Open Access article distributed under the terms of the Creative Commons Attribution Non-Commercial License (http://creativecommons.org/ licenses/by-nc/3.0) which permits unrestricted non-commercial use, distribution, and reproduction in any medium, provided the original work is properly cited. negative lymphovascular invasion, negative vertical margin, and unclear involvement of the horizontal margin (Fig. 2D). To prevent esophageal stenosis after ESD treatment, triamcinolone acetonide was injected into the base of the artificial ulcer, and prednisolone was orally administered for 8 weeks. ${ }^{3}$ The ulcer was cured without stenosis 8 weeks later.

Endoscopic resection for lesions within a physiologically narrow space is difficult. The underwater method is reported to be useful for treatment of rectal tumors near the dentate line and cecal tumors in the appendiceal orifice. ${ }^{4,5}$ We found that the underwater method facilitated ESD for the cervical esophageal cancer by securing a good view. To our knowledge, this is the first report of underwater ESD for a cervical esophageal cancer. 

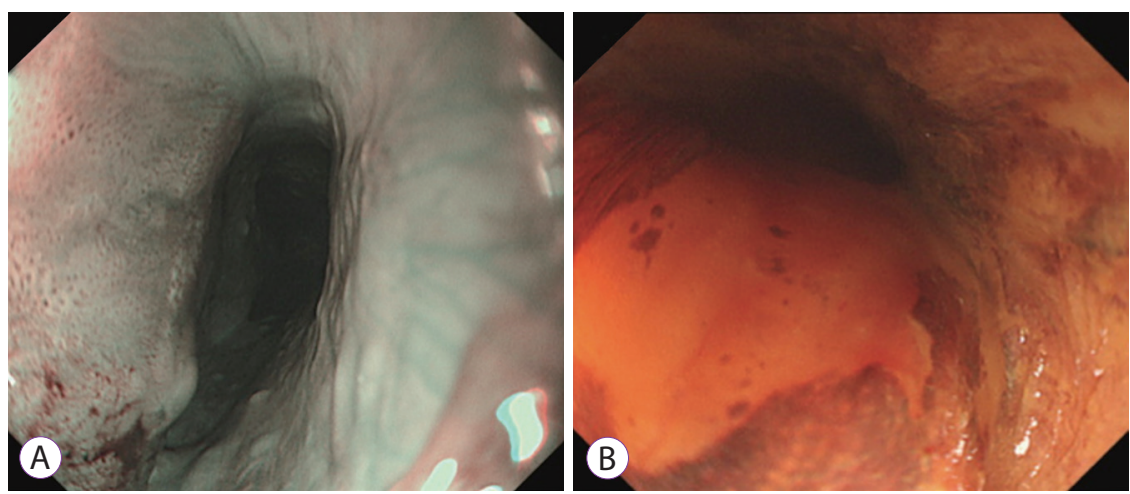

Fig. 1. A superficial esophageal cancer involving the one third of the lumen was found in the cervical esophagus. Due to natural constriction, the lumen was narrow, and endoscopic observation was difficult. (A) Observation via narrow-band imaging revealed the tumor as a brownish area, and tumor blood vessels were dot shaped (B) Lugol staining showed that the tumor was unstained.
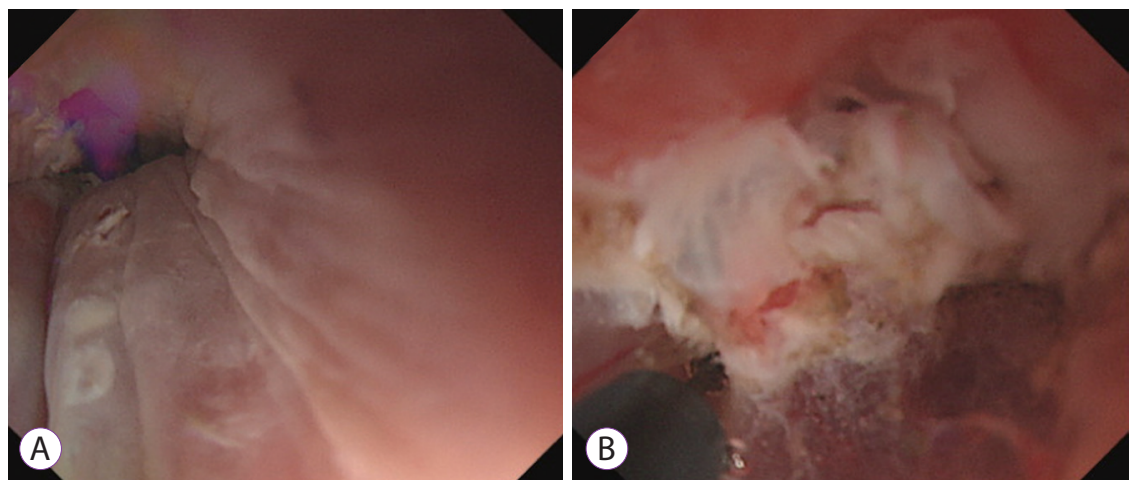

Fig. 2. Underwater endoscopic submucosal dissection was performed on the patient in the
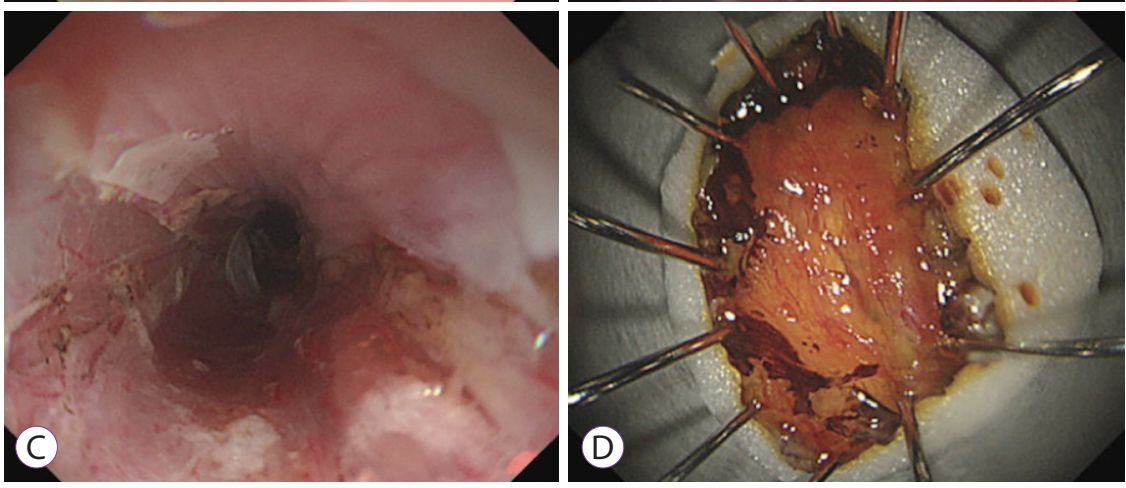
left lateral position with the electrosurgical unit (ERBE VIO 300D; Erbe Elektromedizin, Tübingen, Germany) using the following settings: endocut $Q$, effect 2, duration 3, interval 2; forced coagulation, effect $3,30 \mathrm{~W}$. (A) As the lumen of the esophagus was filled with $0.9 \%$ saline solution using a water pump, the lumen expanded that ensured a visual field and enabled mucosal incision. (B) The submucosal layer floated up, and submucosal dissection proceeded through all the layers without bleeding. (C) The lesion was resected en bloc. (D) Macroscopic image of the resected specimen after Lugol staining showed that the specimen size was $25 \mathrm{~mm}$.

Conflicts of Interest

The authors have no financial conflicts of interest.

\section{Acknowledgements}

My deepest appreciation goes to Dr. Nishikawa whose enormous support and insightful comments were invaluable during the course of this study.

\section{Author Contributions}

Project administration: Kazuhiro Yamamoto

Supervision: Isao Sakaida

Writing-original draft: Sho Sasaki

Writing-review\&editing: Jun Nishikawa

\section{ORCID}

Jun Nishikawa: https://orcid.org/0000-0002-6695-9754

Kazuhiro Yamamoto: https://orcid.org/0000-0001-9576-6997

Isao Sakaida: https://orcid.org/0000-0002-8365-7547

\section{REFERENCES}

1. Binmoeller KF, Weilert F, Shah J, Bhat Y, Kane S. "Underwater" EMR without submucosal injection for large sessile colorectal polyps (with video). Gastrointest Endosc 2012;75:1086-1091.

2. Akutsu D, Suzuki $H$, Narasaka T, et al. Waterjet submucosal dissection of porcine esophagus with the HybridKnife and ERBEJET 2 system: a pilot study. Endosc Int Open 2017;5:E30-E34.

3. Yamaguchi N, Isomoto H, Nakayama T, et al. Usefulness of oral prednisolone in the treatment of esophageal stricture after endoscopic submucosal dissection for superficial esophageal squamous cell carcinoma. Gastrointest Endosc 2011;73:1115-1121.

4. Ishaq S, Kuwai T. Rectal polyp reaching the dentate line: underwater EMR without submucosal lift. VideoGIE 2017;2:53-54.

5. Iacopini F, Gotoda T, Montagnese F, Andrei F, Saito Y. Underwater endoscopic submucosal dissection of a nonpolypoid superficial tumor spreading into the appendix. VideoGIE 2017;2:82-84. 